\title{
Predicting the Outcomes of Subjects With Severe Community- Acquired Pneumonia Using Monocyte Human Leukocyte Antigen-DR
}

\author{
Yugang Zhuang MD, Wenjie Li MD, Huiqi Wang MD, Hu Peng MD, Yanqing Chen MD, \\ Xiangyu Zhang MD PhD, Yuanzhuo Chen MD, and Chengjin Gao MD PhD
}

\begin{abstract}
BACKGROUND: The objective was to study the level of monocyte-human leukocyte antigen-DR (mHLA-DR), an immune function-related biomarker, at $24 \mathrm{~h}$ after admission, to predict the outcomes of subjects with severe pneumonia. METHODS: Subjects with severe community-acquired pneumonia $(n=102)$ were included in the study. Blood samples were collected from each subject $24 \mathrm{~h}$ after admission. Data regarding age, sex, $\mathrm{P}_{\mathrm{aO}_{2}} / \mathrm{F}_{\mathrm{IO}_{2}}$, comorbidities, occurrence of altered mental status, bacteremia, septic shock, Acute Physiology and Chronic Health Evaluation (APACHE) II score, and Sequential Organ Failure Assessment (SOFA) score within the first $\mathbf{2 4}$ h; the highest temperature within $24 \mathrm{~h}$ after admission; mechanical ventilation usage; timing of antibiotic therapy; ICU stay; and 28-d survival were collected. Expression of mHLA-DR was measured by flow cytometry. RESULTS: APACHE II score and SOFA score were significantly higher $(P<.001)$, whereas the mHLA-DR expression was significantly lower $(P<.001)$ in the non-survivors than in the survivors. The outcomes at day 28 after admission were significantly associated with the APACHE II score $(P=.002$, odds ratio $[O R]=1.27,95 \%$ CI $1.10-1.48)$, the SOFA score $(P=.003$, OR $=1.52,95 \%$ CI 1.15-2.00 $)$, and mHLA-DR level $(P=.01$, OR $=0.91,95 \%$ CI 0.85-0.98), as shown by logistic regression. The area under the receiver operating characteristic curve was 0.877 (95\% CI 0.81-0.94, $P<.001), 0.862$ (95\% CI 0.79-0.93, $P<.001)$, and 0.781 (95\% CI 0.69-0.87, $P<.001)$ for APACHE II score, SOFA score, and the mHLA-DR expression, respectively. The optimal threshold for mHLA-DR level was $27.2 \%$. Kaplan-Meier survival analysis showed that subjects with mHLA-DR $\geq 27.2 \%$ had significantly better outcomes compared with $<27.2 \%$ level $(P<.001, \log$ rank test, hazard ratio $=0.963,95 \%$ CI $0.94-0.99)$. CONCLUSIONS: mHLA-DR may be a reliable biomarker that can predict the outcomes of patients with severe community-acquired pneumonia, and $27.2 \%$ may be the cut-off value to predict the outcomes. Key words: severe pneumonia; mHLA-DR; single time point; prognosis. [Respir Care 2015;60(11):1635-1642. @ 2015 Daedalus Enterprises]
\end{abstract}

\section{Introduction}

Currently, $10 \%$ of patients with pneumonia require treatment in the ICU, and the mortality rate of such patients is

\footnotetext{
Mr Zhuang, Mr Peng, Mr Yanqing Chen, and Mr Yuanzhuo Chen are affiliated with the Emergency Department, Shanghai Tenth People's Hospital, Tongji University, School of Medicine, Shanghai, China. Ms Li, Ms Wang, Mr Zhang, and Mr Gao are affiliated with the Intensive Care Unit, Shanghai Tenth People's Hospital, Tongji University, School of Medicine, Shanghai, China
}

This study was supported by National Natural Science Foundation of China Grant 81000024 and Shanghai Municipal Health Bureau Grant 03.02.12.015. The authors have disclosed no conflicts of interest. as high as $30-40 \% .^{1}$ Thus, early identification and management of patients with severe pneumonia is important. The consensus guidelines on the management of community-acquired pneumonia in adults issued by the Infectious Diseases Society of America/American Thoracic Society

\footnotetext{
The first 2 authors contributed equally to this work.

Correspondence: Chengjin Gao, No. 301 Middle Yanchang Road, Shanghai 200072, China. Tel.: 86-15801985172. E-mail: chengjingao2003@126.com. Yuanzhuo Chen, No.301 Middle Yanchang Road, Shanghai 200072, China. Tel.: 86-18917684098. E-mail: chenyuanzhuo021@hotmail.com.
}

DOI: $10.4187 /$ respcare. 03953 
in $2007^{2}$ developed the diagnostic criteria of severe pneumonia, which recommended that such patients should be treated in the ICU. However, to date, no simple and universally accepted biomarker is available for the prediction of the outcomes of patients with severe pneumonia in clinical practice.

Several studies over the past few years have demonstrated that monocyte human leukocyte antigen-DR (mHLA-DR) is a potential biomarker in predicting the outcomes of subjects with certain diseases (including sepsis, severe pancreatitis, non-small cell lung cancer, cirrhosis, and secondary infection), ${ }^{3-8}$ although some studies have contrasting results. ${ }^{9-11}$ The immune system plays an important role in the development, progression, and prognosis of pneumonia, ${ }^{12}$ which suggests that this widely accepted immune function-related biomarker may predict outcomes in patients with severe pneumonia. Until now, no studies have investigated the prognosis prediction value of mHLA-DR in patients with severe pneumonia. Only a few studies have shown that the expression of mHLA-DR is down-regulated in subjects with pneumonia. ${ }^{13-15}$

Compared with previous studies, which dynamically measured mHLA-DR level at different time points for different diseases, ${ }^{5-8}$ the present study investigated the expression of mHLA-DR at $24 \mathrm{~h}$ after admission. This study measured the outcomes in subjects with severe pneumonia on day 28 and evaluated the feasibility of using mHLA-DR measured at $24 \mathrm{~h}$ in predicting the prognosis of such subjects.

\section{Methods}

\section{Subjects}

In this prospective study, subjects with severe community-acquired pneumonia who were treated in the emergency ICU of the Shanghai Tenth People's Hospital were included, and the data were analyzed prospectively. The inclusion criteria were: patients who (1) were transferred to the ICU for treatment within $24 \mathrm{~h}$ and (2) met the diagnostic criteria of severe community-acquired pneumonia. The diagnosis of severe pneumonia was performed according to the guidelines issued by the Infectious Diseases Society of America/American Thoracic Society in 20072; patients with one of the major criteria or 3 or more of the secondary criteria were considered to be suffering from severe pneumonia. The major criteria for diagnosis include: patients who (1) require invasive mechanical ventilation and (2) require vasopressor treatment due to septic shock. The secondary criteria for diagnosis include patients with the following conditions: (1) breathing frequency $\geq 30$ breaths/min; (2) oxygenation index $\left(\mathrm{P}_{\mathrm{aO}_{2}} / \mathrm{F}_{\mathrm{IO}_{2}}\right) \leq 250$; (3) multilobar infiltrates (>2 lobes); (4) altered mental status/disorientation; (5)

\section{QUICK LOOK}

\section{Current knowledge}

Currently, $10 \%$ of patients with pneumonia require treatment in the ICU, and the mortality rate is $30-40 \%$. The early identification and management of patients with severe pneumonia are important. Consensus guidelines on the management of community-acquired pneumonia in adults recommend that these patients be treated in the ICU. However, to date, no simple and universally accepted biomarker is available for the prediction of the outcomes of patients with severe pneumonia.

\section{What this paper contributes to our knowledge}

Immune dysfunction was common in subjects with severe pneumonia. Predicting the outcomes of subjects with severe community-acquired pneumonia using monocyte-human leukocyte antigen-DR level at $24 \mathrm{~h}$ after admission appeared to be feasible and effective. Monocyte-human leukocyte antigen-DR level $<27 \%$ at $24 \mathrm{~h}$ after admission predicted poor outcomes in these subjects.

azotemia (blood urea nitrogen $\geq 20 \mathrm{mg} / \mathrm{dL}$ ); (6) leukopenia (white blood cells $<4.0 \times 10^{3} / \mathrm{mL}$ ); (7) thrombocytopenia (platelet count $<100 \times 10^{3} / \mathrm{mL}$ ); (8) hypothermia (temperature $<36{ }^{\circ} \mathrm{C}$ ); and (9) need for aggressive resuscitation due to hypotension.

Patients were excluded if they fulfilled any of the following criteria: (1) age $>90 \mathrm{y}$ or $<14 \mathrm{y}$; (2) diagnosis of other severe infection; (3) severe dysfunction in other organs; (4) malignancy or autoimmune diseases; (5) treatment with immune-related medicines (adrenal corticosteroids, calcineurin inhibitors, alkylating agents, antimetabolites, antibodies, levamisole, interleukin, interferon, transfer factor, thymosin, etc) before admission to ICU; (6) treatment with radiochemotherapy or cardiopulmonary resuscitation; or (7) patient or family refusal to participate in the study.

\section{Clinical Methods}

Etiological examinations were performed in all subjects who were included in the study. Standard treatments, such as oxygen therapy, organ function support, nutritional support, and symptomatic treatments, were performed for all of the included subjects according to the recommendations of the Infectious Diseases Society of America/American Thoracic Society. ${ }^{2,16}$ A blood sample was collected $24 \mathrm{~h}$ after admission. Data such as age, sex, $\mathrm{P}_{\mathrm{aO}_{2}} / \mathrm{F}_{\mathrm{IO}_{2}}$, comor- 
bidities, occurrence of altered mental status, bacteremia, septic shock, Acute Physiology and Chronic Health Evaluation (APACHE) II score, and Sequential Organ Failure Assessment (SOFA) score within the first $24 \mathrm{~h}$ were collected. Follow-up data for the subjects for the highest temperature within the first $24 \mathrm{~h}$, utilization of mechanical ventilation, timing of antibiotic therapy, ICU stay, and 28-d survival of the subjects were collected. Death was diagnosed according to the clinical death standard, namely the occurrence of cardiorespiratory arrest. Follow up and data collection were performed by trained investigators. The clinicians who were in charge of the treatment of subjects were blinded to the collected data. The present study was approved by the Ethics Committee of the Shanghai Tenth People's Hospital. Written informed consent was obtained from all subjects or their family members before entering the study.

\section{Sample Collection and Processing}

Collection of Whole Blood Samples. A whole blood sample was collected from each subject with severe pneumonia $24 \mathrm{~h}$ after transfer to the ICU. The blood was collected into an ethylenediaminetetraacetic acid anticoagulant tube and stored at room temperature. The blood collection was performed by trained eligible medical officers under the supervision of quality control investigators.

Measuring the Expression of mHLA-DR With a Flow Cytometer. After blood collection, staining and fixation were performed within $24 \mathrm{~h} ; 30 \mu \mathrm{l}$ of anti-human leukocyte antigen-DR (HLA-DR) fluorescein isothiocyanate (eBioscience, 11-9952) and $20 \mu \mathrm{l}$ of CD14-PE (BD Pharmingen, 555398) were added to the tube. Murine antihuman monoclonal antibodies (IgG2a-PE [BD Pharmingen] and IgG2a-fluorescein isothiocyanate [eBioscience]) were used as the isotype control; anti-coagulant blood (50 $\mu \mathrm{l})$ was then placed at room temperature in the dark; $2 \mathrm{~mL}$ of a 10-fold dilution of $1 \times$ lysing solution (LSB03, MultiSciences Biotech, Hangzhou, China) was added; and the mixture was again placed at room temperature in the dark. After the mixture turned translucent, it was centrifuged at $236 \mathrm{~g}$ for $5 \mathrm{~min}$, and the supernatant was discarded. The cells were resuspended, and $2 \mathrm{~mL}$ of phosphate buffer solution was added. After centrifugation at $236 \mathrm{~g}$ for $5 \mathrm{~min}$, the supernatant was discarded. Then $0.3 \mathrm{~mL}$ of $1 \%$ paraformaldehyde was added, and the mixture was stored at $4{ }^{\circ} \mathrm{C}$ until measurement using a flow cytometer (BD FACS Canto Flow Cytometer II 338960, BD Biosciences, San Jose, California) within 24 h. CD14 ${ }^{+}$ cells were determined to be monocytes.

\section{Data Processing and Statistical Analysis}

Results of mHLA-DR examined by flow cytometry were expressed as the percentage of HLA-DR-positive monocytes in all of the monocytes and were divided into 2 groups, survivors and non-survivors, according to the outcomes at day 28 after admission. An independent $t$ test was used to compare the differences in the quantitative data (including age, hospital stay, timing of antibiotic therapy, temperature, $\mathrm{P}_{\mathrm{aO}} / \mathrm{F}_{\mathrm{IO}}$, APACHE II score, SOFA score, and mHLA-DR level) between the 2 groups, and a chisquare test was used to compare the differences in the qualitative data (including sex, etiology, comorbidities, altered mental status, bacteremia, septic shock, and mechanical ventilation). Binary logistic regression estimated the odds ratios (ORs), and the corresponding 95\% CI values reflected the association between the outcomes at day 28 after the admission and APACHE II score, SOFA score, or mHLA-DR level in subjects with severe pneumonia; the receiver operating characteristic curve was also used to estimate the predictive value of these 3 parameters. The optimal threshold was determined by sensitivity and specificity (Youden's index). Kaplan-Meier survival curves were used to define the optimal threshold for survival in both groups. All of the statistical analyses were performed using SPSS 19.0 software. Quantitative data (age, temperature, $\mathrm{P}_{\mathrm{aO}} / \mathrm{F}_{\mathrm{IO}_{2}}$, APACHE II score, SOFA score, expression of mHLA-DR, timing of antibiotic therapy, and ICU stay) were expressed as mean $\pm \mathrm{SD}$, whereas qualitative data (sex, etiology, comorbidities, altered mental status, bacteremia, septic shock, and mechanical ventilation) were expressed as frequencies (percentages). $P<.05$ was considered statistically significant.

\section{Results}

\section{General Characteristics of the Subjects}

A total of 102 subjects with severe pneumonia were successfully followed up, and the completed data were used for the statistical analyses. Tables 1 and 2 show the general characteristics of the subjects, scores, and treatment in the survivors and non-survivors, respectively. There were statistically significant differences in terms of the number of malnourished subjects $(P=.02)$, number of subjects with altered mental status $(P=.001)$, number of subjects with septic shock $(P=.001)$, APACHE II score $(P<0.001)$, SOFA score $(P<0.001)$, timing of antibiotic therapy $(P<0.001)$, and ICU stay $(P<0.001)$ between the survivors and non-survivors. The other features were not significantly different between these 2 groups. 
Table 1. General Characteristics of Subjects

\begin{tabular}{|c|c|c|c|c|}
\hline & Survivors $(n=58)$ & Non-Survivors $(n=44)$ & $P$ & Total $(N=102)$ \\
\hline Age & $27-89(66.24 \pm 13.13)$ & $37-89(70.39 \pm 12.14)$ & .11 & $27-89(68.00 \pm 12.82)$ \\
\hline Sex & & & .72 & \\
\hline Male & $35(60.3)$ & $25(56.8)$ & & $60(58.8)$ \\
\hline Female & $23(39.7)$ & $19(43.2)$ & & $42(41.2)$ \\
\hline \multicolumn{5}{|l|}{ Etiology } \\
\hline Bacterium & $44(75.9)$ & $36(81.8)$ & .63 & $80(78.4)$ \\
\hline Virus & $0(0.0)$ & $0(0.0)$ & NA & $0(0.0)$ \\
\hline Fungi & $26(44.8)$ & $25(56.8)$ & .32 & $51(50)$ \\
\hline Atypical pathogens & $0(0.0)$ & $0(0.0)$ & NA & $0(0.0)$ \\
\hline Physical or chemical & $0(0.0)$ & $0(0.0)$ & NA & $0(0.0)$ \\
\hline Not detected & $3(5.2)$ & $5(11.4)$ & .29 & $8(7.8)$ \\
\hline \multicolumn{5}{|l|}{ Comorbidities } \\
\hline Type 2 diabetes & $7(12.1)$ & $6(13.6)$ & $>.99$ & $13(12.7)$ \\
\hline Coronary heart disease & $17(29.3)$ & $17(38.6)$ & .40 & $34(33.3)$ \\
\hline Hypertension & $29(50.9)$ & $27(61.4)$ & .32 & $56(55.4)$ \\
\hline Malnourished & $6(10.3)$ & $13(29.5)$ & $.02 *$ & $19(18.6)$ \\
\hline Other infection & $26(44.8)$ & $18(40.9)$ & .84 & $44(43.1)$ \\
\hline Temperature, ${ }^{\circ} \mathrm{C}$ & $36.70-41.00(38.06 \pm 1.02)$ & $36.70-40.10(37.92 \pm 1.01)$ & .50 & $36.70-41.00(38.00 \pm 1.01)$ \\
\hline $\mathrm{P}_{\mathrm{aO}_{2}} / \mathrm{F}_{\mathrm{IO}_{2}}$ & $211.33-555.00(370.44 \pm 93.60)$ & $212.33-613.33(344.83 \pm 90.95)$ & .17 & $211.33-613.33(359.40 \pm 92.89)$ \\
\hline Altered mental status & $24(41.4)$ & $33(75)$ & $.001 *$ & $57(55.9)$ \\
\hline Bacteremia & $30(51.7)$ & $20(45.5)$ & .56 & $50(49.0)$ \\
\hline Septic shock & $5(8.6)$ & $16(36.4)$ & $.001 *$ & $21(20.6)$ \\
\hline \multicolumn{5}{|c|}{$\begin{array}{l}\text { Subjects were assigned to the survivors or non-survivors depending on the outcomes on day } 28 \text { after admission. Data are presented as counts (percentage) except for age, temperature, and } \mathrm{P}_{\mathrm{aO}} / \mathrm{F}_{\mathrm{IO}_{2}} \text {, } \\
\text { presented as range (mean } \pm \mathrm{SD} \text { ). } \\
\begin{aligned} * P<.05 \text { was considered statistically significant between the } 2 \text { groups. } \\
\mathrm{NA}=\text { not available }\end{aligned}\end{array}$} \\
\hline
\end{tabular}

Table 2. Scores and Treatment

\begin{tabular}{|c|c|c|c|c|}
\hline & Survivors $(n=58)$ & Non-Survivors $(n=44)$ & $P$ & Total $(N=102)$ \\
\hline APACHE II score & $3-25(13.29 \pm 7.09)$ & $10-42(26.61 \pm 8.68)$ & $<.001^{*}$ & $3-42(19.04 \pm 10.22)$ \\
\hline SOFA score & $2-13(4.48 \pm 2.76)$ & $3-16(9.11 \pm 3.66)$ & $<.001 *$ & $2-16(6.48 \pm 3.91)$ \\
\hline Mechanical ventilation & $37(63.8)$ & $35(79.5)$ & .12 & $72(70.6)$ \\
\hline Timing of antibiotic therapy, $d$ & $2-26(11.52 \pm 5.09)$ & $2-17(7.30 \pm 4.27)$ & $<.001 *$ & $2-26(9.70 \pm 5.18)$ \\
\hline ICU stay, d & $5-22(12.29 \pm 4.34)$ & $3-22(8.36 \pm 5.11)$ & $<.001 *$ & $3-19(8.94 \pm 4.25)$ \\
\hline \multicolumn{5}{|c|}{ 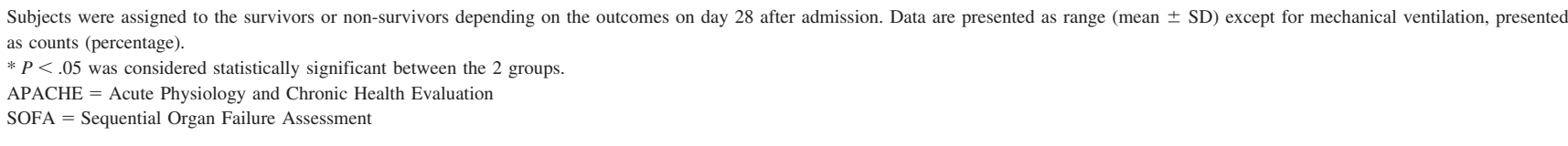 } \\
\hline
\end{tabular}

\section{Outcomes of Subjects With Severe Pneumonia and Association With the APACHE II Score and/or mHLA-DR}

The APACHE II score and SOFA score were significantly higher in the non-survivors than in the survivors $(P<.001$, independent $t$ test, Fig. 1). The mHLA-DR level was significantly lower in the non-survivors than in the survivors $(P<.001$, independent $t$ test, Fig. 1). The outcomes at day 28 after admission were significantly as- sociated with the APACHE II score (13.29 \pm 7.09 and $26.61 \pm 8.68$ for the survivors and non-survivors, respectively; $P=.002$, OR $=1.27,95 \%$ CI $1.10-1.48$, binary logistic regression), the SOFA score $(4.48 \pm 2.76$ and $9.11 \pm 3.66$ for the survivors and non-survivors, respectively; $P=.003, \mathrm{OR}=1.52,95 \% \mathrm{CI} 1.15-2.00$, binary logistic regression), and mHLA-DR level (31.26 \pm 12.10 and $17.89 \pm 12.10$ for the survivors and non-survivors, respectively; $P=.01, \mathrm{OR}=0.91,95 \%$ CI $0.85-0.98$, binary logistic regression). However, the association be- 

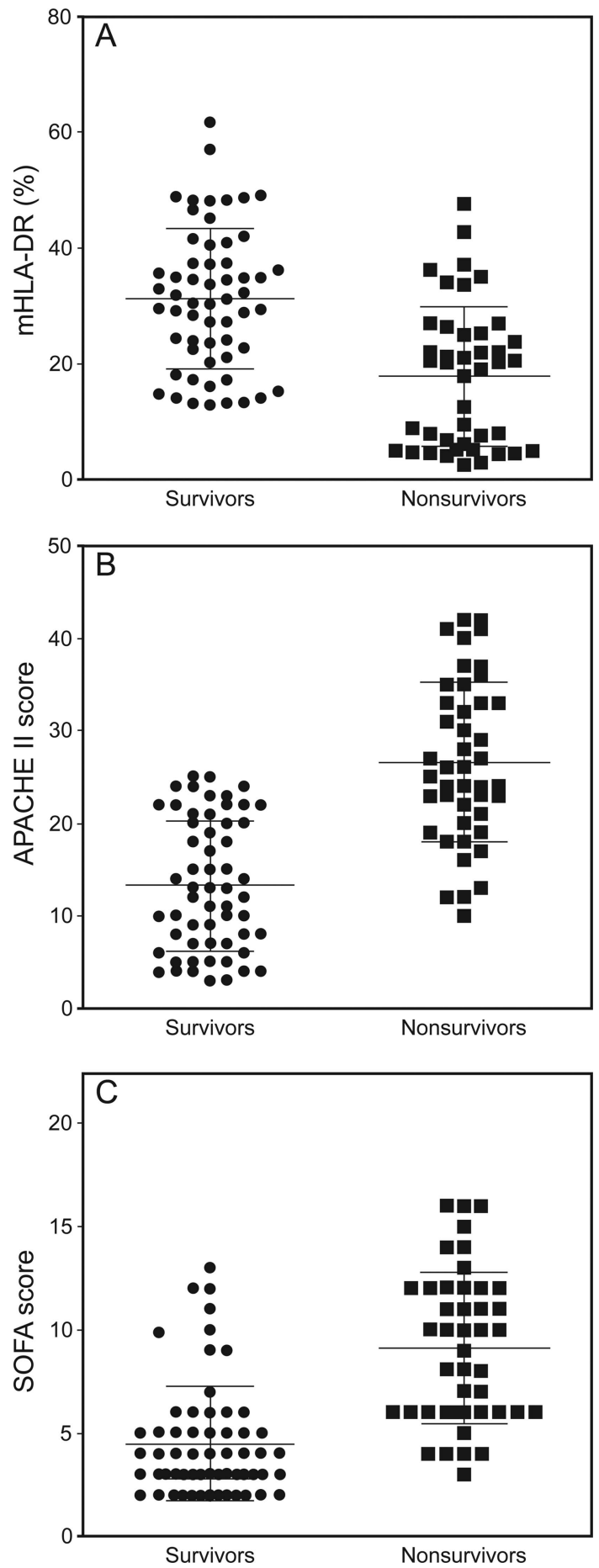

Fig. 1. Subjects with severe pneumonia were divided into survivors ( $n=58)$ and nonsurvivors $(n=44)$ according to the outcomes at $28 \mathrm{~d}$ after admission. A: A flow cytometer was used to evaluate the expression of monocyte human leukocyte antigen-DR (mHLA-DR) $24 \mathrm{~h}$ after admission. The Acute Physiology and Chronic Health Evaluation (APACHE) II score (B) and Sequential Organ Failure Assessment (SOFA) score (C) within $24 \mathrm{~h}$ after admission were used for the statistical analysis. An independent $t$ test was used to compare the 2 groups. Center lines denote the mean, whereas upper and lower lines show SD. $P<.001$ between groups. tween the outcomes at day 28 after submission and the number of malnourished subjects $(P=.22, \mathrm{OR}=0.32$, 95\% CI 0.052-1.96, binary logistic regression), number of subjects with altered mental status $(P=.11, \mathrm{OR}=0.27$, 95\% CI 0.052-1.35, binary logistic regression), or number of subjects with septic shock $(P=.25, \mathrm{OR}=0.28,95 \% \mathrm{CI}$ $0.033-2.46$, binary logistic regression) was not significant.

\section{Determination of the Predictive Value of APACHE II Score and mHLA-DR Expression}

The area under the receiver operating characteristic curve was 0.877 (95\% CI 0.81-0.94, $P<.001), 0.862(95 \%$ CI $0.79-0.93, P<.001)$, and 0.781 (95\% CI $0.69-0.87$, $P<.001)$ for the APACHE II score, SOFA score, and mHLA-DR expression, respectively, suggesting that all of these parameters had predictive values (Fig. 2A). According to Youden's index (sensitivity was $84.1 \%$, and specificity was $58.6 \%$ ), $27.2 \%$ was the optimal threshold for mHLA-DR. Thus, the expression of mHLA-DR in 20 subjects $(34.5 \%)$ in the survivors $(n=58)$ and $37(84.1 \%)$ in the non-survivors $(n=44)$ was below the threshold value. Kaplan-Meier survival analysis, which was performed using the threshold of mHLA-DR, showed that subjects with mHLA-DR $\geq 27.2 \%$ had significantly better outcomes than those with mHLA-DR $<27.2 \%(P<.001, \log$ rank test, hazard ratio $=0.963,95 \%$ CI $0.94-0.99$, Fig. 2B).

\section{Discussion}

In the present study, flow cytometry analysis was used to evaluate the expression of mHLA-DR at a single time point, $24 \mathrm{~h}$ after admission, and revealed that subjects with severe pneumonia had different degrees of immune suppression. The mHLA-DR level at $24 \mathrm{~h}$ after admission was significantly associated with the outcomes of these subjects, which may suggest its feasibility to predict outcomes in patients with severe pneumonia (area under the curve $=0.781$ ), and $27.2 \%$ was the best threshold for prediction of the outcomes of the subjects (sensitivity of $84.1 \%$ and specificity of $58.6 \%$ ).

Currently, pneumonia still remains one of the major causes of death globally, despite advances in treatment. ${ }^{17}$ Early diagnosis of severe pneumonia can enable patients to receive immediate medical attention by transferral to the ICU for further treatment, ${ }^{18}$ and appropriate early intervention may improve their outcomes. ${ }^{19}$ However, blindly depending on clinical presentations, routine examinations, and scoring systems may substantially reduce the accuracy of predicting the outcomes of severe pneumonia in patients, delaying treatment. Considering the significant role of immunity in severe pneumonia and the reversibility of immune suppression, ${ }^{12,20}$ we hypothesized that the measurement of an immune-related biomarker at a single time 

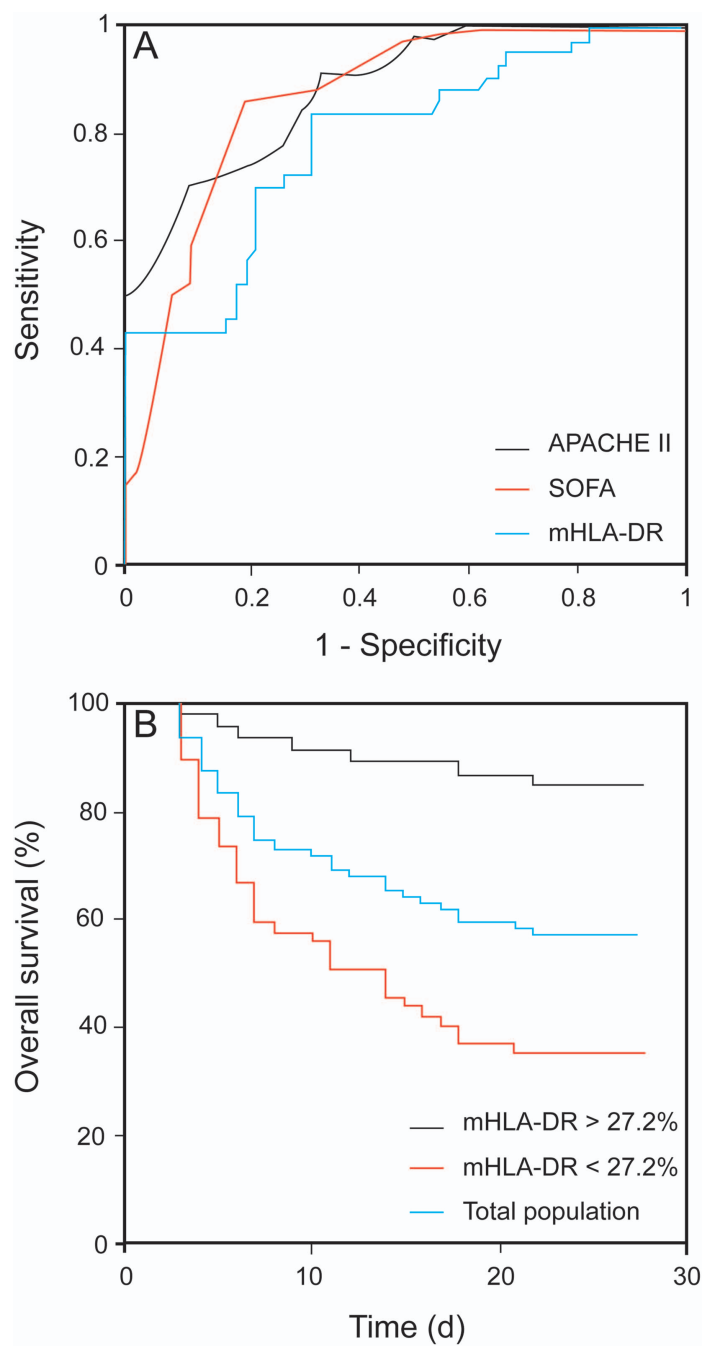

Fig. 2. Value of monocyte-human leukocyte antigen-DR (mHLADR) in predicting the outcomes at day 28 in subjects with severe pneumonia. A: Area under the receiver operating characteristic curve was $0.781,0.877$, and 0.862 for the mHLA-DR expression, Acute Physiology and Chronic Health Evaluation (APACHE) II score, and Sequential Organ Failure Assessment (SOFA) score, respectively. B: Kaplan-Meier survival analysis using the optimal threshold of mHLA-DR (27.2\% optimum according to Youden's index).

point might help clinicians to predict severe pneumonia with poor outcomes that should be treated in the ICU. The present study confirmed the importance of mHLA-DR in predicting outcomes of subjects with severe infections.

HLA-DR is a major histocompatibility antigen-II expressing at the highest level in human beings and mainly distributed in monocytes, B lymphocytes, and endothelial cells. The primary function of HLA-DR is to present peptide antigens that activate $\mathrm{T}$ helper cell responses and lead to the production of antibodies against the same peptide antigen, and its expression has been shown to be regulated by the sum of the effects of multiple mediators, such as interferon- $\gamma$, interleukin-10, corticoids, and cat- echolamines, ${ }^{21}$ which may make its level fluctuate with those mediators in the process and enable it to be a potential marker for immunosuppression in critically ill patients. Expression of HLA-DR is reduced during immune suppression following the hyperinflammatory period, and vice versa. The recommendation by Volk et al ${ }^{22}$ suggests that patients with mHLA-DR $<30 \%$ should be considered as immunosuppresed, and this has been widely accepted. Generally, the HLA-DR level in patients with better outcomes of sepsis recovers in 1 week after administration. 6,23 Infection may be aggravated or uncontrolled in patients with immunosuppression, sometimes even causing death. ${ }^{24}$ Meisel et $\mathrm{al}^{20}$ and Schefold et $\mathrm{al}^{25}$ demonstrated that awareness of the levels of mHLA-DR may help in guiding immunomodulatory therapy, shortening mechanical ventilation time and the duration of hospital stay. In recent years, several studies have shown that mHLA-DR is a valuable factor that can predict the outcomes of subjects with sepsis, severe pancreatitis, non-small cell lung cancers, cirrhosis, and secondary infections ${ }^{3-8}$; some studies concluded otherwise, as the investigators suggested that mHLA-DR is not an independent factor that affects the outcomes of subjects and pointed out the limitations of flow cytometer examinations. ${ }^{9-11}$ The immune system plays an important role in the development and progression of pneumonia, and mHLA-DR is a widely accepted biomarker that can reflect the immune functions. ${ }^{12} \mathrm{~A}$ few studies have shown that the expression of mHLA-DR is down-regulated in pneumonia subjects, ${ }^{13-15}$ although there has been no study that has determined the importance of mHLA-DR in predicting the prognosis of severe pneumonia in patients. We believe that it is worth investigating its feasibility and value in predicting the prognosis of severe pneumonia in patients. Because the times for measuring the mHLA-DR level and the parameters to evaluate the prognosis of subjects were different in previous studies, the findings of the present study provide valuable evidence for future studies investigating the value of mHLA-DR to predict the outcomes of subjects with severe pneumonia as well as using this biomarker in treating patients with severe pneumonia in clinical practice.

In the present study, the mHLA-DR level of subjects with severe pneumonia was detected at a single time point, which was $24 \mathrm{~h}$ after admission, for the following reasons. (1) It takes $24 \mathrm{~h}$ for clinicians to fully understand the disease conditions of patients and to determine the initial intervention. (2) Payen et al ${ }^{26}$ compared subjects with single organ failure with those with multiple organ failure and found that $24 \mathrm{~h}$ could be the earliest turning point for mHLA-DR to recover. Delayed recovery of mHLA-DR level could significantly affect the mortality rate and secondary infection rate in such patients. (3) Severe pneumonia has an acute onset and rapid progression; thus, clinicians need to decide the specific treatment as early as 


\section{Outcomes in Severe Community-Acquired Pneumonia}

possible to improve the outcomes of the patients. (4) Measuring the level at one single time point could facilitate the clinical application and explanation. Monneret et $\mathrm{al}^{27}$ found that the expression of mHLA-DR in the survivors and non-survivors with septic shock was not significantly different until days 3 and 4 . We speculated that the difference of the time of expression of the biomarker in our study could be affected by the different diseases investigated; however, the optimal time for measuring mHLA-DR expression in patients with severe pneumonia must be further investigated.

Patients with disease that had a tremendous impact on immune status were excluded from the study, and the comorbidities that may affect the immune function are listed in Table 1.28-31 Only the number of malnourished subjects displayed a significant difference between the survivors and non-survivors. However, considering the limited number of malnourished subjects and limited association with mortality as shown by logistic regression, it could be deduced that severe community-acquired pneumonia was the main factor that caused the changes of mHLA-DR and mortality.

After measuring the mHLA-DR level at $24 \mathrm{~h}$ after admission, we also found that the mHLA-DR level in subjects with severe pneumonia was generally below 30\% $(25.49 \%)$. This value was significantly lower in the nonsurvivors compared with the survivors, suggesting that immune suppression is a common condition in the early stage in severe pneumonia, and the suppressive degree is associated with poorer outcomes of subjects. The threshold (cut-off value) was determined to be a $27.2 \%$ mHLA-DR level, and the subjects with an mHLA-DR level above or below this value had significantly different outcomes. Because $27.2 \%$ is close to $30 \%$ (the value that has been widely accepted), the findings of the present study showed that immunity plays a crucial role in the prognosis of subjects with severe pneumonia, and mHLA-DR is a reliable factor that reflects the immune functions of patients.

The findings of the present study also determined the predictive value of the APACHE II score and SOFA score for outcomes at day 28 in subjects with severe pneumonia. We found that the mHLA-DR level at $24 \mathrm{~h}$ after admission could predict the outcomes in subjects with severe pneumonia, with the area under the curve slightly lower than that of the APACHE II score and SOFA score. In a study conducted in subjects with sepsis, Perry et $\mathrm{a}^{32}$ found that the value of the mHLA-DR level was greater than that of the APACHE II score in predicting outcomes. We believe that the difference observed is due to differences in the disease type, sampling time, and parameters used to evaluate the outcomes. In addition, both APACHE II and SOFA tend to evaluate the overall function of patients, whereas mHLA-DR focuses on the immune function of patients at a given time point. The study suggests that the use of
mHLA-DR levels to evaluate the immune function of patients, in addition to using the APACHE II score or SOFA score, could be a better strategy for early identification of the disease and/or for ensuring early admittance of patients with possible poor outcomes to the ICU for specific treatments.

There are several limitations in the present study. (1) The sample size was relatively small. Further multi-center studies with a larger sample size are needed to validate our findings. (2) The time from disease onset to admission should be recorded and adjusted. (3) Other biomarkers should be also analyzed and compared with mHLA-DR to select the best predictive biomarker. (4) Dynamic observation of mHLA-DR should be further carried out. In future studies, measures should be taken to avoid these limitations.

\section{Conclusions}

Immune dysfunction is very common in patients with severe pneumonia. Predicting the outcomes of patients with severe community-acquired pneumonia with mHLA-DR level at $24 \mathrm{~h}$ after admission may be feasible and effective. An mHLA-DR level $<27.2 \%$ at $24 \mathrm{~h}$ after admission may predict poor outcomes in patients.

\section{REFERENCES}

1. Ewig S, Woodhead M, Torres A. Towards a sensible comprehension of severe community-acquired pneumonia. Intensive Care Med 2011; 37(2):214-223.

2. Mandell LA, Wunderink RG, Anzueto A, Bartlett JG, Campbell GD, Dean NC, et al. Infectious Diseases Society of America/American Thoracic Society consensus guidelines on the management of community-acquired pneumonia in adults. Clin Infect Dis 2007;44(Suppl 2):S27-S72.

3. Gouel-Chéron A, Allaouchiche B, Guignant C, Davin F, Floccard B, Monneret G, et al. Early interleukin-6 and slope of monocyte human leukocyte antigen-DR: a powerful association to predict the development of sepsis after major trauma. PLoS One 2012;7(3):e33095.

4. Genel F, Atlihan F, Ozsu E, Ozbek E. Monocyte HLA-DR expression as predictor of poor outcome in neonates with late onset neonatal sepsis. J Infect 2010;60(3):224-228.

5. Lin ZQ, Guo J, Xia Q, Yang XN, Huang W, Huang ZW, Xue P. Human leukocyte antigen-DR expression on peripheral monocytes may be an early marker for secondary infection in severe acute pancreatitis. Hepatogastroenterology 2013;60(128):1896-1902.

6. Wu JF, Ma J, Chen J, Ou-Yang B, Chen MY, Li LF, et al. Changes of monocyte human leukocyte antigen-DR expression as a reliable predictor of mortality in severe sepsis. Crit Care 2011;15(5):R220.

7. Berry PA, Antoniades CG, Carey I, McPhail MJ, Hussain MJ, Davies ET, et al. Severity of the compensatory anti-inflammatory response determined by monocyte HLA-DR expression may assist outcome prediction in cirrhosis. Intensive Care Med 2011;37(3):453460.

8. Lukaszewicz AC, Grienay M, Resche-Rigon M, Pirracchio R, Faivre V, Boval B, Payen D. Monocytic HLA-DR expression in intensive care patients: interest for prognosis and secondary infection prediction. Crit Care Med 2009;37(10):2746-2752. 


\section{Outcomes in Severe Community-Acquired Pneumonia}

9. Ploder M, Pelinka L, Schmuckenschlager C, Wessner B, Ankersmit HJ, Fuerst W, et al. Lipopolysaccharide-induced tumor necrosis factor $\alpha$ production and not monocyte human leukocyte antigen-DR expression is correlated with survival in septic trauma patients. Shock 2006;25(2):129-134.

10. Cazalis, Friggeri A, Cavé L, Demaret J, Barbalat V, Cerrato E, et al. Decreased HLA-DR antigen associated invariant chain(CD74) mRNA expression predicts mortality after septic shock. Crit Care 2013; 17(6):R287.

11. Huang A, Zhang B, Wang B, Zhang F, Fan KX, Guo YJ. Increased CD14 (+) HLA-DR (-/low) myeloid-derived suppressor cells correlate with extrathoracic metastasis and poor response to chemotherapy in non-small cell lung cancer patients. Cancer Immunol Immunother 2013;62(9):1439-1451.

12. Mizgerd JP. Acute lower respiratory tract infection. N Engl J Med 2008;358(7):716-727.

13. Chalk K, Meisel C, Spies C, Volk T, Thuenemann K, Linneweber J, et al. Dysfunction of alveolar macrophages after cardiac surgery and postoperative pneumonia?: an observational study. Crit Care 2013; 17(6):R285.

14. Simon PM, Delude RL, Lee M, Kong L, Guzik LJ, Huang DT, et al. Duration and magnitude of hypotension and monocyte deactivation in patients with community-acquired pneumonia. Shock 2011;36(6): 553-559.

15. Polk HC, Naziri W, McCurry TM. Immunologic responses to pneumonia. Am J Surg 2000;179(2):41-44.

16. American Thoracic Society, Infectious Diseases Society of America. Guidelines for the management of adults with hospital-adquired, ventilaror-associated, and healthcare-associated pneumonia. Am J Respir Crit Care Med 2005;171(4):388-416.

17. Guan X, Silk BJ, Li W, Fleischauer AT, Xing X, Jiang X, et al. Pneumonia incidence and mortality in Mainland China: systematic review of Chinese and English literature, 1985-2008. PLoS One 2010;5(7):e11721.

18. Renaud B, Santin A, Coma E, Camus N, Van Pelt D, Hayon J, et al. Association between timing of intensive care unit admission and outcomes for emergency department patients with community-acquired pneumonia. Crit Care Med 2009;37(11):2867-2874.

19. Que YA, Lazar H, Wolff M, François B, Laterre PF, Mercier E, et al. Assessment of panobacumab as adjunctive immunotherapy for the treatment of nosocomial Pseudomonas aeruginosa pneumonia. Eur J Clin Microbiol Infect Dis 2014;33(10):1861-1867.
20. Meisel C, Schefold JC, Pschowski R, Baumann T, Hetzger K, Gregor $\mathrm{J}$, et al. Granulocyte-macrophage colony-stimulating factor to reverse sepsis-associated immunosuppression: a double-blind, randomized, placebocontrolled multicenter trial. Am J Respir Crit Care Med 2009;180(7):640-648.

21. Monneret G, Venet F, Pachot A, Lepape A. Monitoring immune dysfunctions in the septic patient: a new skin for the old ceremony. Mol Med 2008;14(1):64-78.

22. Volk HD, Reinke P, Krausch D. Monocyte deactivation: rationale for a new therapeutic strategy in sepsis. Intensive Care Med 1996; 22(Suppl 4):S474-S481.

23. Hotchkiss RS, Monneret G, Payen D. Sepsis-induced immunosuppression: from cellular dysfunctions to immunotherapy. Nat Rev Immunol 2013;13(12):862-874.

24. Cabrera-Perez J, Condotta SA, Badovinac VP, Griffith TS. Impact of sepsis on CD4 T cell immunity. J Leukoc Biol 2014;96(5):767-777.

25. Schefold JC, von Haehling S, Corsepius M, Pohle C, Kruschke P, Zuckermann $\mathrm{H}$, et al. A novel selective extracorporeal intervention in sepsis: immunoadsorption of endotoxin, interleukin 6, and complement-activating product 5a. Shock 2007;28(4):418-425.

26. Payen D, Faivre V, Lukaszewicz AC, Villa F, Goldberg P. Expression of monocyte human leukocyte antigen-DR in relation with sepsis severity and plasma mediators. Minerva Anestesiol 2009;75(9): 484-493.

27. Monneret G, Lepape A, Voirin N, Bohé J, Venet F, Debard AL, et al. Persisting low monocyte human leukocyte antigen-DR expression predicts mortality in septic shock. Intensive Care Med 2006;32(8): 1175-1183.

28. Pedicino D, Liuzzo G, Trotta F, Giglio AF, Giubilato S, Martini F, et al. Adaptive immunity, inflammation, and cardiovascular complications in type 1 and type 2 diabetes mellitus. J Diabetes Res 2013; 2013:184258

29. Onat A, Dönmez I, Karadeniz Y, Cakır H, Kaya A. Type-2 diabetes and coronary heart disease: common physiopathology, viewed from autoimmunity. Expert Rev Cardiovasc Ther 2014;12(6):667-679.

30. Rabinovitch M, Guignabert $C$, Humbert M, Nicolls MR. Inflammation and immunity in the pathogenesis of pulmonary arterial hypertension. Circ Res 2014;115(1):165-175.

31. Iliakis D, Kressig RW. The relationship between malnutrition and immune. Ther Umsch 2014;71(1):55-61.

32. Perry SE, Mostafa SM, Wenstone R, Shenkin A, McLaughlin PJ. Is low monocyte HLA-DR expression helpful to predict outcome in severe sepsis?. Intensive Care Med 2003;29(8):1245-1252. 